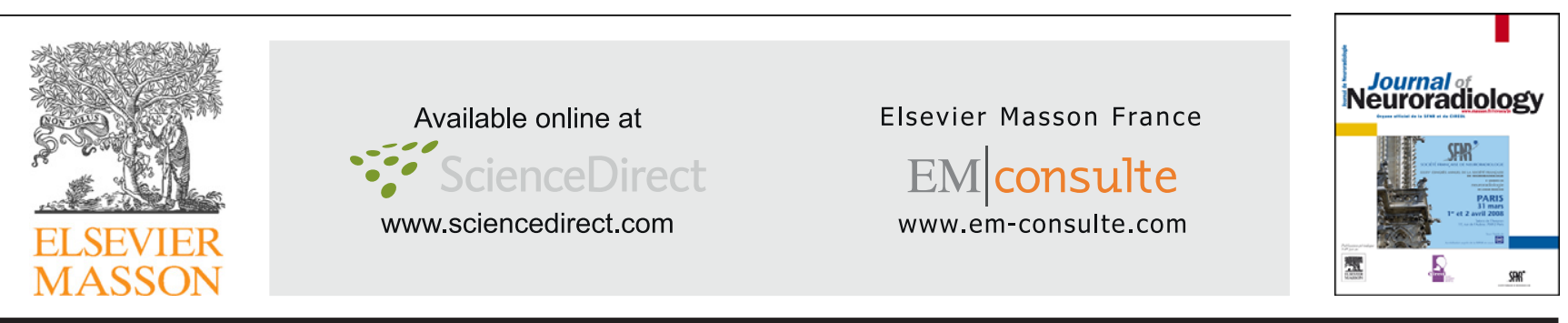

ORIGINAL ARTICLE

\title{
Methodologies to assess blood flow in cerebral aneurysms: Current state of research and perspectives
}

\section{Approches méthodologiques de l'étude des flux dans les anévrismes intracrâniens : état de la recherche et perspectives}

\author{
L. Augsburger ${ }^{a, *, b}$, P. Reymond ${ }^{a}$, E. Fonck ${ }^{a, b}$, Z. Kulcsar ${ }^{b}$, M. Farhat ${ }^{c}$, \\ M. Ohta ${ }^{d}$, N. Stergiopulos ${ }^{a}$, D. A. Ruifenacht ${ }^{b}$
}

a Laboratory of Hemodynamics and Cardiovascular Technology, École polytechnique fédérale de Lausanne, Ecublens, STI IB12 LHTC 1, A1 1241, Station 15, 1015 Lausanne, Vaud, Switzerland

b Neurointerventional Division, Clinical Neurosciences Department, University Hospital of Geneva, Geneva, Switzerland

c Laboratory of Hydraulic Machines, École polytechnique fédérale de Lausanne, Lausanne, Switzerland

${ }^{\mathrm{d}}$ Intelligent Fluid Systems Division, Institute of Fluid Science, Tohoku University, Sendai, Japan

\section{KEYWORDS \\ Cerebral aneurysms; \\ Blood flow \\ assessment; \\ Particle image \\ velocimetry; \\ Computational fluid \\ dynamics}

\begin{abstract}
Summary With intracranial aneurysms disease bringing a weakened arterial wall segment to initiate, grow and potentially rupture an aneurysm, current understanding of vessel wall biology perceives the disease to follow the path of a dynamic evolution and increasingly recognizes blood flow as being one of the main stakeholders driving the process. Although currently mostly morphological information is used to decide on whether or not to treat a yet unruptured aneurysm, among other factors, knowledge of blood flow parameters may provide an advanced understanding of the mechanisms leading to further aneurismal growth and potential rupture. Flow patterns, velocities, pressure and their derived quantifications, such as shear and vorticity, are today accessible by direct measurements or can be calculated through computation. This paper reviews and puts into perspective current experimental methodologies and numerical approaches available for such purposes. In our view, the combination of current medical imaging standards, numerical simulation methods and endovascular treatment methods allow for thinking that flow conditions govern more than any other factor fate and treatment in cerebral aneurysms. Approaching aneurysms from this perspective improves understanding, and while requiring a personalized aneurysm management by flow assessment and flow correction, if indicated.
\end{abstract}

(c) 2009 Elsevier Masson SAS. All rights reserved.
* Corresponding author.

E-mail address: luca.augsburger@epfl.ch (L. Augsburger).

\section{Introduction}

Cerebral aneurysms are pathological dilatations of arteries occurring mainly at bifurcations located in the vicinity of the 
circle of Willis [1]. Under the effect of a single or a combination of factors, the arterial wall is progressively thinned and ballooned out to create an aneurysm. Recognized cofactors contributing to intracranial aneurysm growth and risk of rupture are for example hypertension, atherosclerosis, smoking, excessive alcohol consumption and oral contraceptives. Increased risk exists among patients presenting hereditary deficiencies such as hereditary polycystic kidney disease, Ehlers-Danlos syndrome, Marfan syndrome, fibromuscular dysplasia or family history of aneurysm disease [2-4], and between 7 to $20 \%$ of people who underwent an aneurismal rupture have a relative of first or second degree with a cerebral aneurysm diagnosed [5]. All this indicates that the biology and the resulting life cycle of a specific aneurysm is potentially influenced by a number of factors and coincidences, what allows for comparing the disease with a complex systems, where the variable factors want to be weighed and integrated according to their possibly varying role along the different periods of the aneurysm life cycle, i.e. initiation, growth and rupture.

Clinical studies reveal a prevalence of around $2 \%$ of the Western population having a cerebral aneurysm with a rupture incidence of only about 10 of 100,000 people per year [6]. Aneurysm rupture in these 10 people present mainly under the form of a subarachnoid hemorrhage (SAH), what results in high morbidity and mortality rates for the patients concerned [7], and this even under good medical care conditions. SAH conditions due to a ruptured aneurysm result for obvious reasons in high treatment cost. Treatment of unruptured cerebral aneurysm provides better results and at lower cost, however, not free of risk. With current diagnostic medical imaging methods, revealing increasingly such unruptured aneurysms as an incidental imaging finding, it seems of interest to search for criteria and methods that would allow for optimizing the rupture risk assessment and to weigh such risk against the risk of a potential treatment. To take into account the changing treatment risk, the assessment should be adjustable to the evolving treatment methods and standards. Such an educated approach may allow for reducing the stress conveyed to patients and may provide a useful support to the discussion and decision taking, while avoiding unnecessary treatment or supporting the decision on when and how to treat.

As outlined above, the multiple factors influencing the fate of a cerebral aneurysm require the use of a risk assessment model that perceives the condition as complex. Considering the roles of the different parameters in such a complex system, among others, the group representing biomechanical aspects is of special interest, because methods as developed in engineering and industry could be applied. Measurements or calculations of biomechanical factors include the intra-aneurismal blood flow, the mechanical load on the arterial wall and the peri-aneurismal environment in case appropriate boundary conditions can be defined. The aneurismal shape reflects the wall quality and is the resultant of the aneurysm evolution, and depends from the physiological load, as well as from the contact with surrounding tissues [8], very much alike the riverbed, represents the resultant of the river and the land it runs through. Rupture mainly results from the progressive degeneration and weakening of the three arterial wall constituents, the elastin fibers, the vascular smooth muscles and the collagen fibers. The last to go is the collagen network, which constitutes the major structural element of the aneurismal wall. Contrary to the mainly flow driven degeneration, there have been observation of morphological changes associated with aneurysm wall remodeling representing inflammation or repair attempts.

Through biology, all these mechanisms are influencing the aneurysm wall quality and therefore one could imagine addressing the disease and its evolution by pharmacological therapy influencing degeneration, inflammation or repair [9]. Although yet to explore further, in our view the most promising method to alter the biology of the aneurysm wall is acting on the fluid mechanic parameters, such as blood pressure, flow rates and patterns that may all, individually or in combination, alter the biological response of the wall constituents or of the endothelium, which in turn releases bioactive substances regulating the metabolism and matrix turnover of the aneurismal wall [1]. If acting on the vessel lumen side to implement flow correction through medical devices like coils and stents seems increasingly feasible, acting on the outside of the aneurysm wall is less promising. Modification of the peri-aneurismal environment is currently only possible by applying surgical wrapping methods [10].

Therefore blood flow modulation may constitute and increase its role as an efficient treatment principle, aiming either disconnection from the parent artery flow or restoration of a physiological flow in the parent artery. Neurosurgical treatment performed by placing a metallic clip around the aneurismal neck is closing definitively the aneurismal flow and may change the geometry of the parent artery. This could be seen as a therapy inducing flow modification in the aneurysm and the parent arteries. Although the surgical modality offers the advantage of a direct vessel repair, this is achieved with the effort of an open head surgery associated accordingly with patient discomfort and operative risk [7]. Less discomfort and procedural risk exists with endovascular repair, working minimally invasive through a small arterial puncture site and by using image guided treatment methods [11]. Endovascular treatment methods act by flow modulation in the aneurismal cavity or in the parent vessel. The aneurismal cavity can be filled with coils leading rapidly to aneurismal thrombosis once enough coils have been introduced. Use of stents may act as a scaffold to help keeping coils in an aneurismal cavity in case there is a large aneurysm neck and offering a higher vessel reconstruction success rate, with the stent acting as a scaffold for the vessel wall remodeling. The main difficulties of endovascular treatment are related to difficult access to the target area, intra-operative rupture risk and mainly a currently high peri-operative thromboembolic potential. Based on these facts, the decision for invasive treatment has to balance between the risks of treatment and the risks of natural disease course [12,13].

With this potential in mind, it is believed that stents alone will be able to redirect the flow in the parent artery and slow down the aneurismal flow leading to a thrombus formation, stabilizing the aneurysm without the need to enter and to touch the fragile aneurismal cavity. Prediction of intraaneurismal flow alterations after stent placement could be helpful to foresee the effect and to allow for choosing the most adequate stent capable of inducing alone or together 


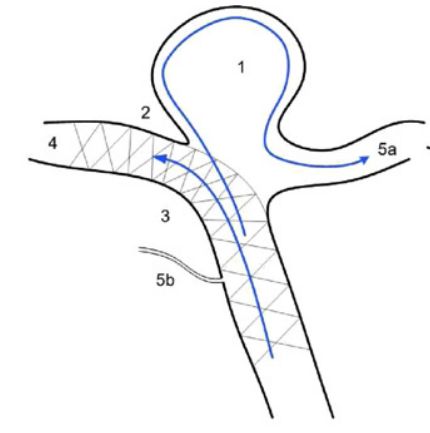

Aneurysm:

1) Cavity: Rupture / Clotting

2) Neck: Growth Matrix

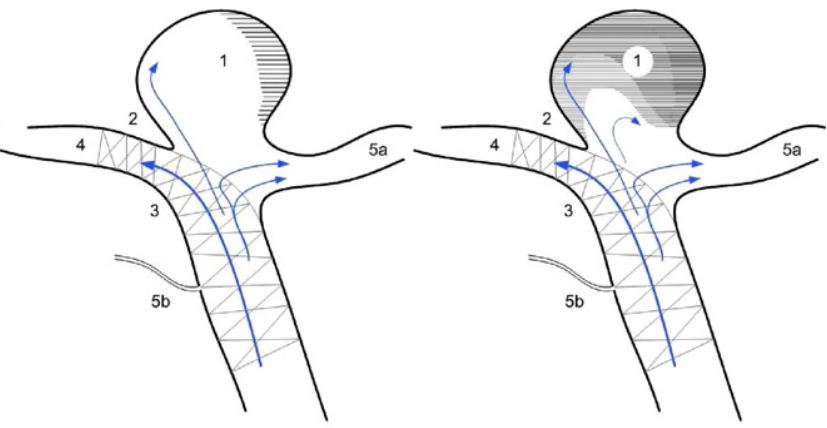

Artery:
3) Wall
4) Parent Artery
5a) Large side branch
5b) Small side branch

Figure 1 Illustration of the progression of clot formation in a bifurcation aneurysm model treated with an intracranial stent as seen in medical procedures.

with additional intra-aneurismal flow modifiers (coils) a stable and safe clot formation (Fig. 1).

Such predictions could empower medical doctors to choose the most appropriate treatment. Medical imaging companies would have to integrate numerical simulation tools and medical device companies would have to develop and produce improved endovascular prostheses - processes that are currently well under way.

In this paper, we present a review of the current state of research involving in vitro experiments and numerical simulations of intra-aneurismal flow in presence of a stent.

\section{Experimental methods developed to assess blood flow changes due to a stent in intracranial aneurysms: evolution of methods and transparent in vitro vascular models (phantoms, replicas)}

One of the objectives of experimental methods is to assess blood flow changes in aneurysms induced by flow diverters such as may be produced by a stent deployed in the parent artery. Assessing the device effect on flow in in vitro vascular phantoms is done to estimate in vivo flow effects to be expected. This effort is aimed mainly at predicting the chance to obtain thrombus formation within an aneurysm after stent placement. The tests may include assessment of effects on flow in the parent artery and its branches. There are quantifiable flow effects, such as values of velocities and pressure and their related parameters, including vorticity (circulation), helicity (rotation), wall shear stress (WSS), and oscillatory shear index among others. Visualization and classification of altered blood flow patterns appears further of interest when it comes to estimating stent efficiency.

The materials of vascular replicas have evolved and spatial resolution of 3D data improved stepwise, giving today possibilities to a range of model production reaching from simple idealized shapes up to complex, patient-specific cerebral aneurysm replicas based on three-dimensional rotational angiography (3D-RA) [14]. Using rapid prototyping (RP) technologies, 3D description of the aneurysm geometry serves to produce models made of transparent materials such as plexiglas (polymethyl-methacrylate), silicone or poly-vinyl-alcohol-hydrogel, (PVA-H). All such models are transparent and compatible with current medical imaging methods. Most used in our practice are silicone and PVA models. The low friction characteristics of PVA-H models and its elasticity close to soft tissue when compared to silicone models allow for more realistic simulation when it comes to study pulsatile flow effects.

The following flow measuring and visualization techniques have allowed for simple visualization up to a detailed mapping of the parameters defining flow within an aneurismal cavity.

\section{Slip stream visualization}

\section{Slip stream visualization without device}

In 1992, flow patterns around human cervical carotid bifurcations were visualized using digital subtraction angiography by injecting contrast agents [15], allowing for direct visualization of flow. The authors hypothesized that high WSS could cause degenerative changes in the endothelial layer, initiating the formation of saccular and fusiform intracranial aneurysms. One year later, by injection of fluorescent particles in the blood stream, Nakatani et al. could visualize spiral blood flows in vivo, both proximal and distal to arterial bifurcations located at the base of rat brains [16]. This study led to hypothesize on aneurysms development, growth, and rupture. Chong et al. compared the flow patterns in a vertebrobasilar artery model using slip stream visualization and MR based flow measurements. Flow profiles were found to be consistent between both techniques [17].

\section{Slip stream visualization with device}

Lieber et al. visualized the pulsatile flow patterns in an experimental flow apparatus using a laser-induced 
fluorescence of rhodamine dye. Various stents exhibiting four different porosities (comprised between 76 and 85\%) were investigated and the results showed the difference of aneurismal vortex reduction in large and small arteries [18]. Using a photochromic dye, Rhee et al. visualized the flow in sidewall and in fusiform aneurysms models, and velocity and WSS changes due to various stents could be calculated. The results reported in these two studies revealed different results with flow reduction being not similar in both kind of models and with stents of various porosities, a less porous stent reducing the intra-aneurismal fluid motion significantly, and in the other, with stent porosity of less significant effect [19].

\section{Particle illumination photography}

Barath et al. studied 20 different stents of various ranges of porosity, permeability and filament diameter and quantified the reduction of the vortex displacement in two different neck-sized sidewall aneurysm models under unsteady flow conditions. Flow patterns were visualized by using glass particles and laser sheet translumination. The method defines a vortex center path line method obtained by sequentially subtracting each image taken, followed by identification of the vortex center and by tracking the vortex center producing a center path line. The conditions were studied without and in presence of different stents [20]. The authors raised the question of stent positioning effects. In a second study [21], the authors studied the intra-saccular flow pattern changes and the vortex velocity reduction induced by a stent in a internal carotid artery model retrieved from human vascular casting.

\section{Particle image velocimetry (PIV)}

PIV is a non-intrusive technique which allows reconstructing the velocity vectors fields in a flowing fluid seeded with particles. This technique, which is widely adopted in a variety of liquid and gas flows, has been used by Lieber et al. to quantify the effects of the strut size of three helical stents on flow [22], and by Canton et al. to measure the intra-aneurysm flow dynamics and mechanical stresses changes resulting from the placement of neuroform stents in bifurcating intracranial aneurysms models [23]. Measurements of the circulation demonstrated a reduction of the vortex magnitude due to the placement of one, two or three stents. PIV was also used by $\mathrm{Yu}$ et al. in sidewall aneurysm models under steady flow conditions, where the Reynolds number varied from 200 to 1600. They noticed that for Reynolds number higher than 700 , a large recirculating vortex would be formed. The insertion of two different stents damped the flow movement, the mean velocity and reduced consequently the WSS [24].

\section{Laser Doppler velocimetry (LDV)}

Other techniques, such as LDV are also used to assess flow and WSS in patient-specific geometries. LDV is an accepted method in velocity measurement investigations based on the cross of two beams of collimated, monochromatic, and coherent laser light in the flow of the fluid being measured.

Validation and comparison with numerical simulations and clinical data without device. Liou et al. compared tridimensional flow fields with computational fluids dynamics (CFD) simulations in idealized, lateral and rigid cerebral aneurysm models and found that the inflow angle into the lateral aneurysm, the maximum WSS acting on the distal lip of the lateral aneurysm and the intra-aneurismal vortical motion increased with decreasing aneurysm size, underlining the potential critical aneurysm geometrical configuration leading to rupture [25]. Tateshima et al. measured and evaluated the alternation and distribution of WSS in two aneurysm models (middle cerebral artery and basilar tip, both with blebs at their domes). They noticed that both aneurysm models blebs were exposed to high WSS, region where the arterial wall is the finest in vivo [26]. Hollnagel et al. used LDV to validate phase-contrast magnetic resonance angiography (PC-MRA) in a patient specific aneurysm model under steady flow conditions. They found only minor differences in velocity field distribution and mean velocity values between both techniques, the accuracy depending on the artery size and the measurement plane positioning [27].

Our group measured the aneurismal flow, qualified and quantified the flow reduction due to the insertion of an endovascular prosthesis using PIV. Fig. 2A to D present the aneurysm flow in a lateral aneurysm placed on a curved parent artery model without $(A$ and $C)$ and with $(B$ and $D)$ a commercial stent (Neuroform II, Boston Scientific, Fremont, CA) under a steady flow of $100 \mathrm{ml} / \mathrm{min}$. The corresponding Reynolds number is 70.2 and the stent effective porosity defined to be the ratio of the free surface area over the stent area is calculated to reach $82 \%$. For this specific case, we quantified the mean velocity magnitude reduction within the aneurysm to reach $69 \%$; in other words, only $31 \%$ of the flow is remaining; $78 \%$ reduction in vorticity.
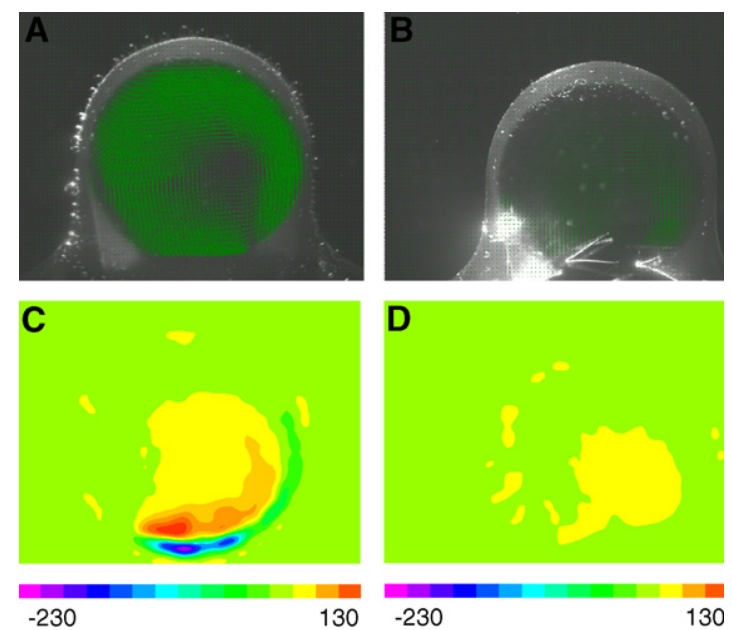

Figure 2 Velocity vector fields without (A) and with a stent (B); vorticity magnitudes without (C) and with stent (D) are presented. 


\section{Prediction of hemodynamical changes due to a stent in aneurysms models using computational fluids dynamics: numerical models}

\section{Simulation without stent}

With the improvement of computer power and the evolution in computational fluid dynamics, numerical simulations gained in speed and precision. Nowadays, an up-to-date laptop or small cluster of computers has enough power to compute the flow in a patient specific aneurismal geometry under unsteady flow conditions within a few hours. The quality of medical images permits the reproduction of the cerebral geometry very precisely, so that intra-aneurismal flow can be simulated accurately. The combination of advanced image processing and geometrical modeling techniques with computational fluid dynamics enhanced the generation of detailed hemodynamical aneurismal flow in patient-specific geometries [14,28]. Cebral et al. developed a CFD pipeline allowing for the computation of the arterial and aneurismal flow based on CTA and 3D rotational angiography image data. The morphological boundaries of the vessel lumen were obtained through 3D reconstruction, segmentation and meshing. Physiological flow boundaries were set based on ultrasound measurements. Subsequent computation provided blood flow patterns, WSS, oscillatory shear and pressure data. Their results enhanced the understanding of intra-aneurismal flow patterns and their relation to aneurismal rupture risk [29]. Four flow pattern categories were defined: simple stable, complex stable, simple unstable and complex unstable. Aneurysms of the first group were less at risk of rupture whereas aneurysms presenting a complex unstable flow were more likely to be associated with rupture. The additional evaluation of the jet type entering into the aneurismal cavity showed it to be a potential indicator of aneurismal rupture. Indeed, geometries aiding the creation of a fluid jet impinging on a small aneurismal wall area belonged mainly to ruptured groups. Conversely, geometries where flow was more diffused and entered less canalized into the aneurysms were less at risk of rupture [30].

Some studies used such CFD simulations to propose flowbased indices for the prediction of aneurismal rupture risk or even the rupture location [31,32]. It has been shown that flow features are strongly dependant on aneurysm geometry. It has been further hypothesized that flow, having a strong relation to geometrical aspects, could be the principal equivalent of a surrogate biomarker of aneurismal rupture [29].

Valencia et al. pointed out the relationship between WSS and aneurysm area index. In fact, by defining the aneurysm surface index as the ratio between the aneurysm surface area and the arterial lumen cross-sectional area, they found a correlation between the mean WSS on the aneurismal sac and the aneurysm surface. Aneurysms presenting a higher surface with low mean WSS were more likely to rupture [33]. Numerical simulations matched to clinical observation allowed to correlate the high fluid-induced WSS with ruptured areas [34].

\section{Simulation with stent}

Due to complex problem of representing a patient-specific geometry and due to the huge computational load numerical simulations are requiring, blood flow was simulated in twodimensional idealized geometries. Ohta et al. investigated the hemodynamical flow patterns in intracranial aneurysms bifurcations models before and after stent placement over a cardiac cycle. As blood was modeled to be a non-Newtonian fluid, the lowered values of peak velocities and WSS due to stent placement allowed to localize the region of increased dynamic viscosity [35].

Numerical simulations were also performed to assess how stents can be optimized to enhance their ability at diverting the flow and, consequently, reduce the risk of rupture. Stent effects on aneurismal flow, WSS, pressure and oscillatory shear were quantified. Aenis et al. [36] investigated the flow patterns in a sidewall aneurysm under physiological pressure and flow conditions. The stent altered the local hemodynamics and substantial differences in flow patterns and pressure values were observed and quantified. Stuhne et al. developed mesh generation tools to manage the difficulties encountered with the meshing of stented aneurysm geometries. More detailed flow patterns description could be provided [37]. Cebral et al. present an adaptive grid embedding technique to manage the mesh difficulties due to scales differences between artery and stent strut sizes [38]. The modeling of stent deployment in a patient-specific geometry is currently achieved by morphing technique. Appanaboyina et al. developed a method that deforms a straight stent model and fits it in a patientspecific geometry. Basically, a cylindrical surface composed of shell elements is generated along the skeleton of the parent vessel of the patient-specific geometry and is considered as an elastic material. This surface is expanded under the action of internal smoothing forces and external attractive forces to the wall. The resulted deformation of the cylindrical surface is performed interactively and the expansion is stopped when most of the points on the cylinder touch the wall [39]. This methodology is successfully used to model patient-specific anatomies with different stents allowing for the exploration of different stent designs efficiency.

Other numerical simulation techniques, such as Lattice-Boltzmann method (LBM), were developed to avoid the mesh generation difficulties encountered with finite element and finite volume methods [40]. An LBMbased clotting model for stented intracranial aneurysms has been developed [41] and a direct clotting metric measuring the percentage of clotting of aneurysm impacted by different stents have been compared with indirect metrics measuring both velocity and WSS reductions inside the aneurysm [42].

Our group measured the aneurismal flow, qualified and quantified the flow reduction due to the insertion of an endovascular prosthesis using the techniques described above. Fig. 3 shows the results of blood flow simulation in a patient specific geometry under unsteady flow conditions. The aneurysm geometry was retrieved using 3D-RA and the volume was processed to be finally meshed. Blood flow was measured in the internal carotid artery of a healthy volunteer using PC-MR technique [43]. This physiological flow waveform was applied at the inlet by defining fully spatial 

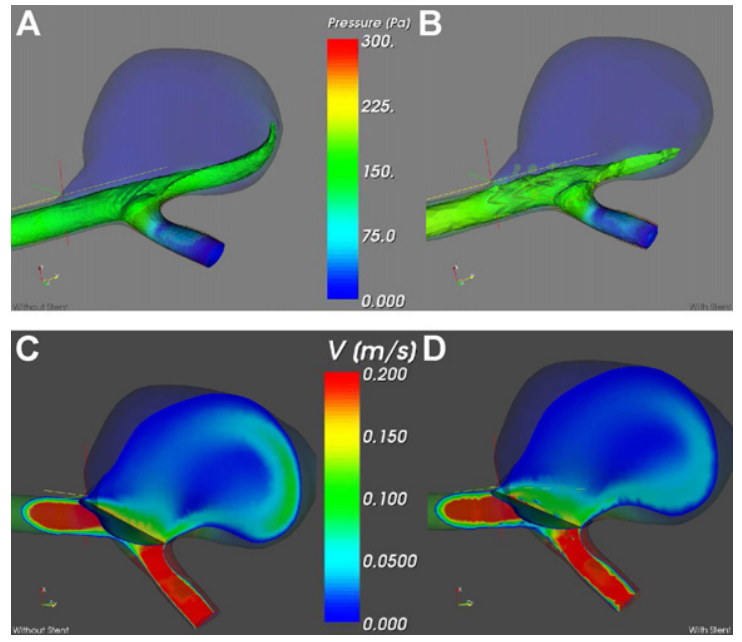

Figure 3 Isovelocity surface $(0.1 \mathrm{~m} / \mathrm{s})$ colored in pressure without device $(A)$ and with device $(B)$, velocity magnitude in cross-sectional and neck planes, without device (C), and with device (D).

and temporal developed velocity profiles (Womersley profiles). A virtual stent was further inserted in the parent artery and flow was computed. The efficiency of the stent to reduce flow and vorticity within the aneurysm was assessed. Although a residual flow remains inside the aneurysm, a reduction of the jet entering the aneurismal cavity can be observed (Fig. 3B). It can be noticed that the Z-like stent, presenting in this case an open-cell design and a rather large porosity of $82 \%$, is still able to reduce somewhat the entry of flow from the parent artery. This reduction is small because stent porosity is high (82\%). Stents currently under development aim at much lower porosities so as to increase much greater flow entry impediment.

In order to demonstrate the clinical value of numerical simulations of the effects of stent on intra-aneurismal flow, the Virtual Intracranial Stenting Challenge (VISC'07, [44] ${ }^{1}$ ) was launched and discussed at the 4th Intracranial Stent Meeting (ICS'07, Kyoto). Its objectives were to compare state-of-the-art numerical approaches for solving flow in a stented human cerebral aneurysm model and to illustrate the ability of academic groups and leading CFD companies in providing a description of changes in aneurismal blood flow patterns for a given stent design. The adopted methodology, as well as simulation parameters, software and hardware choices, simulation times, stent performances were compared. Although some differences in the magnitude of the computed WSS and the velocity field were reported, it was demonstrated that numerical simulations were reproducible among teams. The VISC'07 reinforced the potential role of CFD simulations in providing a description of patient-specific blood flow information in clinical settings, enabling the virtual evaluation of stent performances. The ultimate goal is to use such methods for the selection of an appropriate stent prior to intervention. Also, the same methodology can be used by stent manufacturers for performance analysis and development of novel stent designs.

\section{Discussion/conclusion}

Flow is believed to be a major actor in the initiation, growth and rupture of cerebral aneurysms, hence, the interest for the development of methodologies allowing for the assessment of blood flow within the aneurysm and the flow-driven biomechanical effects.

The modeling of such complex systems must be further improved by including wall motion, wall stress, mechanotransduction at the endothelial and medial cell level and clot formation mechanics.

Due to the complex modeling of patient-specific aneurysm geometries and endovascular prosthesis representations, experimental setups allowed for the understanding of aneurismal hemodynamical effects due to a stent or a coil prior to numerical approaches.

The most common methodologies to assess blood flow in cerebral aneurysm models are PIV and numerical simulations. Patient specific numerical simulations approaches have the potential to become the method of choice for planning, treatment and follow-up decisions. Numerical simulations have proved to be reproducible among teams and to be quite similar to experimental observations and measurements and may therefore become a post-processing path-empowering personalized treatment strategies. However, in order to increase their impact and general acceptance, further efforts are needed to reach agreement between experimental and virtual worlds. Should numerical analysis of blood flow in aneurysms reach the level of being precise and fast enough to be seamlessly integrated with the imaging procedures, physicians would possess a valuable tool for assessing the effects of flow and the impact of their gestures on local hemodynamics in a quantitative way. We may imagine that numerical simulations could be carried out at the moment a patient enters in a hospital and integrated in the medical image acquisition modalities. Results would arrive in time allowing treating physicians to decide on treatment modality. A personalized treatment could be subsequently proposed.

This objective in mind, a sixth framework programme (information society technology, FP6-IST), @neurIST 2 is aiming at developing the required IT for supporting a management that can integrate the complexity of data made available with modern medicine of cerebral aneurysms.

\section{Conflict of interests}

No potential conflict of interests relevant to this article was reported.

\section{Acknowledgments}

Enabled by the Swiss National Research Foundation (Grant Number 3252B0-105735/1) and receiving additional support from the Egon Naef Foundation for in vitro research (Geneva, Switzerland, www.fondation-naef.com), this work was generated in the framework of the @neurIST Project, which is

\footnotetext{
${ }^{1}$ http://www.cilab.upf.edu/visc06/.
}

\footnotetext{
2 www.aneurist.org.
} 
co-financed by the European Commission through the contract no. IST-027703.

We would like to express our best thanks to MrLouis Auer (www.elastrat.com, Geneva, Switzerland) for developing and providing the silicone vascular models.

The authors also wish to warmly thank DrTiziano Binzoni, PhD, Dr Alessandro G. Radaelli, PhD for their precious advices, DrFumio Asakura, MD, and all the technicians from the Hydraulic Machines Laboratory (Imh.epfl.ch, Lausanne, Switzerland) for their support and help in conducting the measurements.

\section{References}

[1] Humphrey JD (2002). Cardiovascular solid mechanics: cells, tissues, and organs. Springer-Verlag, New-York, 757 pp.

[2] Weir BK, et al. Cigarette smoking as a cause of aneurysmal subarachnoid hemorrhage and risk for vasospasm: a report of the Cooperative Aneurysm Study. J Neurosurg 1998;89: 405-11.

[3] Cloft HJ, et al. Prevalence of cerebral aneurysms in patients with fibromuscular dysplasia: a reassessment. J Neurosurg 1998;88:436-40.

[4] Schievink WI, et al. Intracranial aneurysm surgery in EhlersDanlos syndrome Type IV. Neurosurgery 2002;51:607-11 [discussion 611-3].

[5] Cannon Albright LA, et al. A genealogical assessment of heritable predisposition to aneurysms. J Neurosurg 2003;99: 637-43.

[6] Vernooij MW, et al. Incidental findings on brain MRI in the general population. N Engl J Med 2007;357:1821-8.

[7] Wiebers DO, et al. Unruptured intracranial aneurysms: natural history, clinical outcome, and risks of surgical and endovascular treatment. Lancet 2003;362:103-10.

[8] San Millan Ruiz D, et al. The perianeurysmal environment: influence on saccular aneurysm shape and rupture. AJNR Am J Neuroradiol 2006;27:504-12.

[9] Frosen J, et al. Remodeling of saccular cerebral artery aneurysm wall is associated with rupture: histological analysis of 24 unruptured and 42 ruptured cases. Stroke 2004;35: 2287-93.

[10] Kim LJ, Klopfenstein JD, Spetzler RF. Clip reconstruction and sling wrapping of a fusiform aneurysm: technical note. Neurosurgery 2007;61(Suppl. 3):79-80 [discussion 80].

[11] Molyneux AJ, et al. International subarachnoid aneurysm trial (ISAT) of neurosurgical clipping versus endovascular coiling in 2143 patients with ruptured intracranial aneurysms: a randomised comparison of effects on survival, dependency, seizures, rebleeding, subgroups, and aneurysm occlusion. Lancet 2005;366:809-17.

[12] van der Schaaf I, et al. Endovascular coiling versus neurosurgical clipping for patients with aneurysmal subarachnoid haemorrhage. Cochrane Database Syst Rev 2005;4:CD003085.

[13] Rufenacht $D$, et al. Current concepts of endovascular aneurysm treatment, and about the role of stents for endovascular repair of cerebral arteries. Schweiz Arch Neurol Psychiatr 2004;155:348-52.

[14] Cebral JR, et al. Efficient pipeline for image-based patientspecific analysis of cerebral aneurysm hemodynamics: technique and sensitivity. IEEE Trans Med Imaging 2005;24:457-67.

[15] Kim C, et al. In vivo study of flow pattern at human carotid bifurcation with regard to aneurysm development. Acta Neurochir (Wien) 1992;115:112-7.

[16] Nakatani $\mathrm{H}$, et al. In vivo flow visualization of induced saccular cerebral aneurysms in rats. Acta Neurochir (Wien) 1993;122:244-9.
[17] Chong BW, et al. Blood flow dynamics in the vertebrobasilar system: correlation of a transparent elastic model and MR angiography. AJNR Am J Neuroradiol 1994;15:733-45.

[18] Lieber BB, Stancampiano AP, Wakhloo AK. Alteration of hemodynamics in aneurysm models by stenting: influence of stent porosity. Ann Biomed Eng 1997;25:460-9.

[19] Rhee K, Han MH, Cha SH. Changes of flow characteristics by stenting in aneurysm models: influence of aneurysm geometry and stent porosity. Ann Biomed Eng 2002;30:894-904.

[20] Barath K, et al. Influence of stent properties on the alteration of cerebral intra-aneurysmal haemodynamics: flow quantification in elastic sidewall aneurysm models. Neurol Res 2005;27(Suppl. 1):S120-8.

[21] Barath K, et al. Anatomically shaped internal carotid artery aneurysm in vitro model for flow analysis to evaluate stent effect. AJNR Am J Neuroradiol 2004;25:1750-9.

[22] Lieber BB, et al. Particle image velocimetry assessment of stent design influence on intra-aneurysmal flow. Ann Biomed Eng 2002;30:768-77.

[23] Canton G, et al. Flow changes caused by the sequential placement of stents across the neck of sidewall cerebral aneurysms. J Neurosurg 2005;103:891-902.

[24] Yu SC, Zhao JB. A steady flow analysis on the stented and non-stented sidewall aneurysm models. Med Eng Phys 1999;21: 133-41.

[25] Liou TM, Chang WC, Liao CC. LDV measurements in lateral model aneurysms of various sizes. Exp Fluid 1997;23:317-24.

[26] Tateshima S, et al. In vitro measurement of fluid-induced wall shear stress in unruptured cerebral aneurysms harboring blebs. Stroke 2003;34:187-92.

[27] Hollnagel DI, et al. Laser Doppler velocimetry (LDV) and 3D phase-contrast magnetic resonance angiography (PC-MRA) velocity measurements: validation in an anatomically accurate cerebral artery aneurysm model with steady flow. J Magn Reson Imaging 2007;26:1493-505.

[28] Steinman DA, et al. Image-based computational simulation of flow dynamics in a giant intracranial aneurysm. AJNR Am J Neuroradiol 2003;24:559-66.

[29] Cebral JR, Hernandez M, Frangi AF. Computational analysis of blood flow dynamics in cerebral aneurysms from CTA and 3D rotational angiography image data. In: Doblaré AF, Cerrolaza $M$, Rodrigues $\mathrm{H}$, editors. International congress on computational bioengineering. 2003.

[30] Cebral JR, et al. Characterization of cerebral aneurysms for assessing risk of rupture by using patient-specific computational hemodynamics models. AJNR Am J Neuroradiol 2005;26:2550-9.

[31] Ortega HV. Computer simulation helps predict cerebral aneurysms. J Med Eng Technol 1998;22:179-81.

[32] Cebral JR, et al. Blood-flow models of the circle of Willis from magnetic resonance data. J Eng Math 2003;47:369-86.

[33] Valencia A, et al. Blood flow dynamics in patient-specific cerebral aneurysm models: The relationship between wall shear stress and aneurysm area index. Med Eng Phys 2008;30(3):329-40. Epub 2007.

[34] Hassan T, et al. Computational replicas: anatomic reconstructions of cerebral vessels as volume numerical grids at three-dimensional angiography. AJNR Am J Neuroradiol 2004;25:1356-65.

[35] Ohta $M$, et al. Rheological changes after stenting of a cerebral aneurysm: a finite element modeling approach. Cardiovasc Intervent Radiol 2005;28:768-72.

[36] Aenis M, Stancampano AP, Wakhloo AK, Lieber BB. Modeling of flow in a straight stented and nonstented side wall aneurysm model. J Biomech Eng 1997;119(2):206-12.

[37] Stuhne GR, Steinman DA. Finite-element modeling of the hemodynamics of stented aneurysms. J Biomech Eng 2004;126: 382-7. 
[38] Cebral JR, Lohner R. Efficient simulation of blood flow past complex endovascular devices using an adaptive embedding technique. IEEE Trans Med Imaging 2005;24:468-76.

[39] Appanaboyina $S$, et al. Computational fluid dynamics of stented intracranial aneurysms using adaptive embedded unstructured grids. Int J Numerical Methods Fluids 2008;57: 475-93.

[40] Hirabayashi $M$, et al. Characterization of flow reduction properties in an aneurysm due to a stent. Phys Rev E Stat Nonlin Soft Matter Phys 2003;68:021918.

[41] Chopard B, et al. Lattice Boltzmann modeling of thrombosis in giant aneurysms. Int J Mod Phys C 2007;18:712-21.
[42] Chopard B, Ouared R, Rufenacht D. A lattice Boltzmann simulation of clotting in stented aneursysms and comparison with velocity or shear rate reductions. Math Comput Sim 2006;72: $108-12$.

[43] Tateshima S, et al. Intraaneurysmal flow visualization by using phase-contrast magnetic resonance imaging: feasibility study based on a geometrically realistic in vitro aneurysm model. $\mathrm{J}$ Neurosurg 2004;100:1041-8.

[44] Radaelli AG, et al. Reproducibility of haemodynamical simulations in a subject-specific stented aneurysm model - A report on the Virtual Intracranial Stenting Challenge 2007. J Biomech 2008;41(10):2069-81. Epub 2008. 